\title{
A Novel Methodology for Health Hazard and Risk Assessment of Dermal and Inhalation Exposure
}

\author{
Rafeqah Raslan ${ }^{1,2}$, Mimi H. Hassim ${ }^{1 * 2}$, Nishanth G. ChemmangatTUVAlapPIL ${ }^{3}$ and Denny K. S. NG ${ }^{4}$ \\ ${ }^{1}$ School of Chemical and Energy Engineering / Centre of Hydrogen Energy, Universiti Teknologi Malaysia, Johor, Malaysia \\ ${ }^{2}$ Department of Chemical Engineering, Universiti Teknologi MARA, Selangor, Malaysia \\ ${ }^{3}$ Department of Chemical and Environmental Engineering/ Centre of Excellence for Green Technologies, The University of \\ Nottingham Malaysia Campus, Selangor, Malaysia \\ ${ }^{4}$ School of Engineering and Physical Sciences, Heriot-Watt University Malaysia, Putrajaya, Malaysia
}

\begin{abstract}
Household products such as dishwasher products and multipurpose cleaners may contain specific chemical ingredients to meet the consumer needs. However, some of the ingredients may result in skin and respiratory irritation. Thus, a systematic methodology to estimate the extent of hazard and risk for consumers' exposure to the products is needed. In this work, an index-based methodology is presented to estimate the severity of the hazards and risks of the ingredients at during the early stage of product design. Higher score was assigned to the higher potential of hazard and risk, and vice versa. The hazard potential was determined based on hazard classification by the Global Harmonised System (GHS). Risk assessment was performed by considering the Margin of Exposure (MOE) and Risk Characterization Ratio (RCR). To demonstrate the proposed methodology, the dermal and inhalation hazards as well as risks from ingredients used in formulation of liquid detergent were evaluated.
\end{abstract}

\section{Introduction}

Consumers use household products (e.g., bleaching liquid, laundry detergent and toilet cleaner) daily. Such products may consist of various hazardous ingredients that may cause skin and respiratory irritation. For example, exposure to preservatives, which used as antimicrobial ingredients, may lead to skin sensitization and allergic contact dermatitis (Schwensen and Thyssen, 2016). Meanwhile, inhalation of household disinfectants may cause serious lung injuries and even lead to fatality (Park et al., 2014). Due to the abovementioned risks, there is a need to evaluate the potential hazard of such ingredients to the consumers health. However, a comprehensive health hazard assessment of household product ingredients has not been well studied (Wang et al., 2019). Previously, health assessment works were focusing on assessment of household ingredients in a finished product. However, it is noted that hazardous ingredients should be identified at the early stage of product design so that it can be avoided to be included in the final products.

Chemical toxicity distributions (CTD) based on median lethal dose (LD50), lowest observed-adverseeffect level (LOAEL), and no-observed-adverse-effect level (NOAEL) of chemical ingredients are commonly found in material safety data sheet (MSDS) or chemical safety data sheet (CSDS). Note that CTD can be used for chemicals risk assessment, in the absence of toxicity information. The NOAEL has been used to derive the margin of exposure (MOE) to estimate the extent of chemical exposure. Yost et al. (2016) have conducted a health risk assessment of chloroxylenol in liquid hand soap and dishwashing soap. MOE has been reported to estimate the severity of the exposure to chloroxylenol. Alternatively, the Registration, Evaluation, Authorization and Restriction of Chemical (REACH) regulation has established the risk characterization ratio (RCR) as an indicator to predict the potential health risks to consumer. The ratio is calculated by comparing the value of derived no effect level (DNELs) to the estimated exposure levels (McKee et al., 2018).

In this work, a novel systematic methodology which determines the severity level of hazards and risks due to household products ingredients' exposure is proposed. The methodology is used to evaluate the hazards and risks of ingredients during the product formulation design. Besides, such methodology is also used as initial screening tool to reduce the hazards and risks of household products. The scopes of the present work were designed: 1) to collect toxicology data of household products' ingredients; 2) to distinguish the degree of severity of the hazards and risks (low, medium and high) by assigning the respective scores and 3 ) to identify the hazardous ingredient with high scores. A formulation of liquid detergent used for dishwashing was presented as a case study to illustrate the proposed methodology. Two exposure routes of dermal and inhalation were considered.

*Corresponding author: mimi@cheme.utm.my/mimiharyani@utm.my 


\section{Methodology}

\subsection{Hazard Identification}

The Globally Harmonised System of Classification and Labelling Chemicals (GHS, 2013) provides a harmonized basis for globally uniform standard by presenting safety, health, physical and environmental information on hazardous chemical substances. The specified hazards are represented by hazard statements to indicate the degree of severity of the hazard. The advantage of GHS classification is the information provided may facilitate the worldwide chemical user since the hazards have been properly assessed and identified on an international basis. The GHS classification of chemical substances is available in MSDS, CSDS and chemical databases such as PubChem.

\subsection{Hazard Characterization}

The severity of the hazard was determined by assigning a score to distinguish between the low, medium and high degree. The hazardous potential of ingredients that indicated no effect was assigned with a score of 0 , low hazard with a score of 1 , medium hazard with a score of 2 and high hazard with a score of 3 . Table 1 lists the hazard classification for dermal effects while Table 2 shows that of the inhalation effects. The scores assigned to each effect are also presented.

Table 1. Score for dermal exposure

\begin{tabular}{ccc}
\hline $\begin{array}{c}\text { Dermal } \\
\text { effect }\end{array}$ & Score information & Score \\
\hline Acute effect & No effect & 0 \\
& H312: Harmful in contact with skin & 1 \\
& H311: Toxic in contact with skin & 2 \\
H310: Fatal in contact with skin & 3 \\
Skin & H316: Cause mild skin irritation & 1 \\
corrosion / & H315: Cause skin irritation & 2 \\
irritation & H314: Cause severe skin burns & 3 \\
$\begin{array}{c}\text { Skin } \\
\text { sensitization }\end{array}$ & H317: May cause an allergic skin \\
reaction & 3 \\
\hline
\end{tabular}

Table 2. Score for inhalation exposure

\begin{tabular}{|c|c|c|}
\hline $\begin{array}{l}\text { Inhalation } \\
\text { effect }\end{array}$ & Score information & Score \\
\hline \multirow[t]{4}{*}{ Acute effect } & No effect & 0 \\
\hline & H332: Harmful if inhaled & 1 \\
\hline & H331: Toxic if inhaled & 2 \\
\hline & H330: Fatal if inhaled & 3 \\
\hline \multirow[t]{2}{*}{$\begin{array}{l}\text { Respiratory } \\
\text { irritation }\end{array}$} & $\begin{array}{l}\text { H335: May cause respiratory } \\
\text { irritation }\end{array}$ & 1 \\
\hline & $\begin{array}{l}\text { H336: May cause drowsiness or } \\
\text { dizziness }\end{array}$ & \\
\hline $\begin{array}{l}\text { Respiratory } \\
\text { sensitization }\end{array}$ & $\begin{array}{l}\text { H334: May cause allergy or asthma } \\
\text { symptom }\end{array}$ & 3 \\
\hline
\end{tabular}

\subsection{Exposure Assessment}

The exposure assessment was performed to estimate the worst-case exposure scenario from household products' ingredients. The amount, frequency and the concentration of ingredients per usage were taken into account (SCCS, 2012). Two exposure indicators were considered in this work, namely MOE and RCR. The calculation of MOE and RCR are shown in Equation 1 and Equation 2 respectively. Meanwhile, the exposure concentration through dermal $\left(\mathrm{C}_{\mathrm{d}}\right)$ was based on Sanderson et al. (2006) as shown in Equation 3. Concentration through inhalation exposure $(\mathrm{C} i)$ was calculated as shown in Equation 4 following the report by Kim et al., (2018).

$$
\begin{gathered}
M O E=N O A E L / C_{d} \text { or } C_{i} \\
R C R=C_{d} \text { or } C_{i} / D N E L \\
C_{d}=F Q \times C A \times P C \times 10^{3} \times C F \times 10^{-2} \times F T \times D_{a b s} \\
/ \mathrm{BW}
\end{gathered}
$$

$F Q$ is frequency of use ( 3 use/day), $C A$ is the body surface contact area $\left(1680 \mathrm{~cm}^{2}\right), P C$ is product concentration $\left(0.0015 \mathrm{~g} / \mathrm{cm}^{3}\right), C F$ is concentration of ingredients in the formulation (\%) as depicted in Table 4, $F T$ is film thickness on skin $(0.0024 \mathrm{~cm}), D_{a b s}$ is the dermal absorption of the substance $(100 \%)$ and $B W$ is the human body weights of $60 \mathrm{~kg}$ bw (Sanderson et al., 2006).

$$
C_{i}=A \times F Q \times C F \times 10^{-2} \times /(V \times N \times 24)
$$

$A$ is amount per use (5mg), $F Q$ is frequency of use (1.167 use/day) and $C F$ is similar with Equation 3. $V$ is volume of space $\left(15 \mathrm{~m}^{3}\right)$ and $N$ is ventilation rate in $\left(2.5 \mathrm{~h}^{-}\right.$ $\left.{ }^{1}\right)$. The data on $A, F Q, V$ and $N$ were obtained from ConsExpo (2018).

\subsection{Risk Characterization}

The degree of severity of risk was based on the values of MOE and RCR, depending on the availability of the data, that means either NOAEL or DNEL. The minimum value of MOE which is 100 was considered, hence, the value of MOE that is lesser than 100 poses a risk to the consumer (SCCS, 2012). The lesser the value of MOE, the higher the health risks. On the other hand, the minimum value of RCR is 1 , whereby RCR lesser than 1 indicates a safe condition (McKee et al., 2018). The higher value of RCR represents higher risk to consumer.

In this work, a score of 1 was assigned to low risk, thereby indicating no concern of the exposure to the ingredients. Medium risk was assigned a score of 2, thereby reflecting concern on consumer control exposures. The highest score of 3 was allocated to the estimation of high risk, thereby showing high concern and degree of severity of ingredients. The details of the score are presented in Table 3 . 
Table 3. Score for inhalation exposure

\begin{tabular}{cccc}
\hline MOE & RCR & Description & Score \\
\hline MOE $\geq 100$ & RCR $\leq 1$ & Low risk & 1 \\
MOE $<100$ & $\mathrm{RCR}>1$ & Medium risk & 2 \\
$\mathrm{MOE} \leq 1$ & $\mathrm{RCR} \geq 100$ & High risk & 3 \\
\hline
\end{tabular}

\section{Results and Discussion}

The potential hazard and risk of the ingredients contained in liquid detergent formulation (Zhang et al., 2017) was assessed using the proposed methodology. Table 4 presents the ingredients used and its concentration. The detergent formulation presented has used a novel designed solvent where toxicology data such as NOAEL or DNEL was not reported.

Table 4. Formulation of liquid detergent (Zhang et al., 2017)

\begin{tabular}{ccc}
\hline Type & Ingredient & $\begin{array}{c}\text { Concentration } \\
(\mathbf{\% w})\end{array}$ \\
\hline Surfactant & C11-13 Linear alkyl & 28 \\
& $\begin{array}{c}\text { benzene sulfonate (LAS) } \\
\text { C14-15 Alkyl Sulfate } \\
\text { C11-13 Alcohol } \\
\text { ethoxylate (E8) }\end{array}$ & 14 \\
& C16-18 Alkyl N-methyl & 3 \\
glucamide & 2 \\
Enilder & Oleic acid & 3.4 \\
& Citric acid & 5.4 \\
Enzyme & Protease & 1 \\
\hline
\end{tabular}

The hazard score was used to identify the hazardous ingredients in the formulation. The ingredients with high score of 3 were identified as hazardous ingredients. The toxicology information on hazard and the score given are shown in Table 5. No hazard information was found for both dermal and inhalation exposure to alkyl sulfate. Dermal and inhalation exposure to citric acid were shown to be of high hazard. Furthermore, protease was identified to be hazardous due to dermal exposure. Other ingredients in the formulation including oleic acid and linear alkyl benzene sulfonate possessed medium hazard potential. It is remarkable to note that both alcohol ethoxylate and alkyl N-methyl glucamide were found to have no effect in regard to dermal and inhalation exposure.

Table 5. Toxicology information and hazard score

\begin{tabular}{|c|c|c|c|c|}
\hline Ingredient & Dermal & Score & Inhalation & Score \\
\hline LAS & $\begin{array}{c}\text { skin } \\
\text { irritant }\end{array}$ & 2 & No effect & 0 \\
\hline $\begin{array}{l}\text { Alkyl } \\
\text { Sulfate }\end{array}$ & \multicolumn{4}{|c|}{ not available } \\
\hline $\begin{array}{l}\text { Alcohol } \\
\text { ethoxylate }\end{array}$ & $\begin{array}{c}\text { No } \\
\text { effect }\end{array}$ & 0 & No effect & 0 \\
\hline $\begin{array}{l}\text { Alkyl N- } \\
\text { methyl } \\
\text { glucamide }\end{array}$ & $\begin{array}{l}\text { No } \\
\text { effect }\end{array}$ & 0 & No effect & 0 \\
\hline Oleic acid & H315 & 2 & H335 & 1 \\
\hline Citric acid & $\begin{array}{l}\text { H315 } \\
\text { H317 }\end{array}$ & $\begin{array}{l}2 \\
3\end{array}$ & H334 & 3 \\
\hline Protease & $\begin{array}{l}\text { H315 } \\
\text { H317 }\end{array}$ & $\begin{array}{l}2 \\
3\end{array}$ & No effect & 0 \\
\hline
\end{tabular}

The scores obtained from the calculation of MOE and RCR were used to describe the risk characterization as shown in Table 6. No data on NOAEL and DNEL were obtained for alkyl N-methyl glucamide and protease, thus the risk assessment was not performed. Ingredients with high score of 3 indicates the high potential of risk. Based on the result of the scores, no ingredient was found to portray a high potential of risk. All the assessed ingredients showed low risk due to dermal and inhalation exposures during application of the product.

Table 6. Score of risk characterization

\begin{tabular}{llclc}
\hline Ingredient & MOE & Score & RCR & Score \\
\hline LAS & 501.94 & 1 & n.a & n.a \\
Alkyl & 1582.4 & 1 & n.a & n.a \\
Sulfate & 8 & & & \\
Alcohol & n.a & n.a & $2.43 \times 10^{-4}$ & 1 \\
ethoxylate & n.a & n.a & $2.43 \times 10^{-4}$ & 1 \\
Oleic acid & n.a & n.a & 0.5722 & 1 \\
& n.a & n.a & 0.0031 & 1 \\
Citric acid & n.a & n.a & 0.0082 & 1 \\
& n.a & n.a & $3.10 \times 10^{-5}$ & 1 \\
\hline
\end{tabular}

n.a not available

Exposure to citric acid via dermal and inhalation were found to cause severe allergy to consumer. Inhaling citric acid may cause respiratory mucous membrane irritation (TOXNET, 2019) and lead to breathing difficulties. However, the risk was at low potential as shown from the score obtained for risk characterization. It is worth to note that ingredient with high hazard may not necessarily result to similar extent or severity of risk. For protease, no risk assessment has been performed due to limitation of data. However, the hazard assessment showed high hazard potential via dermal exposure. Hence, it is recommended that protease to undergo further testing and analysis. Other ingredients were shown to possess medium and low potential of hazard and risk. The result proof that the proposed methodology is able to distinguish clearly the level of severity of the hazard and risk. The role and relation between hazard and risk are discussed.

\section{Conclusion}

The novel scoring methodology is proposed to assess the health hazard and risk to household products' ingredients exposure. The allocation of score can reveal the severity level of hazard and risk. With the score, the hazardous ingredient in the product formulation can be identified. Furthermore, the proposed methodology can be utilized by the manufacturers at the early design stage to screen out the ingredients with high potential of hazard and risk. Thus, the final product formulation is designed with safer and healthier ingredients.

\section{Acknowledgements}

The authors would like to thank the Institution of Engineers Malaysia, Universiti Teknologi MARA and Ministry of Education Malaysia for the financial support. 


\section{References}

ConsExpo; "Consumer Exposure"

(2018) https://consexpoweb.nl/.

CPISI; "Cleaning Product Ingredient Safety Initiative Database"(2018).

https://www.cleaninginstitute.org/CPISI/

ECHA; "European Chemicals Agency. Registration dossier" (2018).

https://echa.europa.eu/registration-dossier

GHS; "United Nations Globally Harmonised System of Classification and Labelling of Chemicals (GHS, Rev. 5)" Part 3, Health Hazards (2013).

Kim, J. H., T. Kim, H. Yoon, A. Jo, D. Lee, P. Kim, and J. Seo; "Health risk assessment of dermal and inhalation exposure to deodorants in Korea", Sci Total Environ, 625, 1369-1379(2018)

https://doi.org/10.1016/j.scitotenv.2017.12.282.

Mckee, R. H., R. Tibaldi, M. D. Adenuga, J. C. Carrillo, and A. Margary; "Assessment of the Potential Human Health Risks from Exposure to Complex Substances in Accordance with REACH Requirements". "White Spirit" as a Case Study". Regul Toxicol Pharmacol, 92, 439-457 (2018). https://doi.org/10.1016/j.yrtph.2017.10.0.

Park, D.U., J.H. Leem, K.M. Lee, H.K. Lim, Y.Y. Choi, J.J. Ahn, S.Y. Lim, J.I. Park, K.H. Choi, N.R. Lee, H.J. Jung, J.S. Ha, and D.Y. Paek; "Exposure Characteristics of Familial Cases of Lung Injury Associated With the use of Humidifier Disinfectants," Environ. Health. 13, 70 (2014) https://doi.org/10.1186/1476-069X-13-70

Sanderson, H., J. L. Counts, K. L. Stanton, and R. I Sedlak; "Exposure and Prioritization--Human Screening Data and Methods for High Production Volume Chemicals in Consumer Products: Amine Oxides A Case Study," Risk Anal, 26 (6), 1637-57(2006) https://doi.org/10.1111/j.15396924.2006.00829.x.

SCCS; The SCCS's Notes of Guidance for the Testing of Cosmetic Substances and Their Safety Evaluation 8th Revision Scientific Committee on Consumer Safety, (2012)

Schwensen, J.F. and J.P. Thyssen; "Contact Allergy to Preservatives- Is the European Commission a Commendable Risk Manager?", Cosmetics, 3, 1-8 (2016) https://doi.org/10.3390/cosmetics3030029

TOXNET; "Toxicology Data Network" (2019) https://toxnet.nlm.nih.gov/cgibin/sis/search/a?dbs+hsdb: @ term+@,DOCNO+911

Wang, Z., D. Dinh, W. C. Scott, E. S. Williams, M. Ciarlo, P. Deleo, and B. W. Brooks; "Critical Review and Probabilistic Health Hazard Assessment of Cleaning Product Ingredients in All-Purpose Cleaners, Dish Care Products, and Laundry Care Products," Environ Int, 125, 399-417(2019)

https://doi.org/10.1016/j.envint.2019.01.079

Yost, L. J., J. D. Rodricks, D. Turnbull, P. C. Deleo, J. F. Nash, A. Quinones-Rivera, and P. A. Carlson; "Human Health Risk Assessment of Chloroxylenol in Liquid Hand Soap and Dishwashing Soap Used By Consumers and
Health-Care Professionals", Regul Toxicol Pharmacol,80,116-24(2016) https://doi.org/10.1016/j.yrtph.2016.06.003.

Zhang, L., K. Y. Fung, X. Zhang, H. K. Fung, and K. M. $\mathrm{Ng}$; "An Integrated Framework for Designing Formulated Products, " Computers \& Chemical Engineering,107, 6176(2017)

https://doi.org/10.1016/j.compchemeng.2017.05.014 